\title{
Pneumologie und Sport: Ein ambulantes Ausdauertraining mit sportmedizinischer Anleitung als effektive nicht-medikamentöse Therapie- maßnahme in der Pneumologie - eine Projektstudie
}

\author{
Pneumology and Sports: An Outpatient Endurance Training with Sports Medical Guidance as an Effective \\ Non-pharmacological Therapy in Pneumology - a Feasibility Study
}

Autoren

Institute

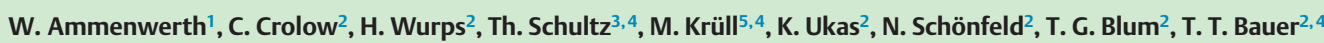

${ }^{1}$ Pneumologische Gemeinschaftspraxis Schmitz/Wiegemann/Ammenwerth, Bochum

${ }^{2}$ Klinik für Pneumologie, Lungenklinik Heckeshorn, Helios Klinikum Emil von Behring, Berlin

${ }^{3}$ Pneumologische Gemeinschaftspraxis Lichterfelde, Berlin

${ }^{4}$ PneumoCare $\mathrm{GmbH}$, Berlin

${ }^{5}$ SMS-Sportmedizin Berlin, Berlin eingereicht 16.11 .2015 akzeptiert nach Revision 2.2.2016

\section{Bibliografie}

DOI http://dx.doi.org/

10.1055/s-0042-102299

Online-Publikation: 8.3.2016

Pneumologie 2016; 70: 314-319

(c) Georg Thieme Verlag KG

Stuttgart · New York

ISSN 0934-8387

Korrespondenzadresse

\section{Dr. Wilhelm Ammenwerth}

Pneumologische

Gemeinschaftspraxis Schmitz/

Wiegemann/Ammenwerth

Kortumstr. 100

44787 Bochum

ammenwerth@lungenarzt-in-

bochum.de

\section{Zusammenfassung \\ V}

Hintergrund: In der Rehabilitation stehen im Fachgebiet der Inneren Medizin muskuläre Ausdauerbelastungen im Vordergrund des sporttherapeutischen Handelns. Leider ist das ambulante Rehabilitations- und Sportangebot für viele pneumologische Patienten begrenzt, und Lungensportgruppen sind noch nicht flächendeckend vorhanden. So wurde ein ambulantes Ausdauersportprogramm mit sportmedizinischer Begleitung als sporttherapeutische Maßnahme in der Pneumologie entwickelt und auf Effektivität überprüft.

Methode: In diese Projektstudie wurden konsekutiv 31 Patienten ( $50 \pm 15$ Jahre) mit verschiedenen pneumologischen Erkrankungen eingeschlossen. Der ambulanten sporttherapeutischen Intervention (12-wöchiges aerobes Ausdauersportprogramm mit $\geq 3$ Einheiten à $20-60 \mathrm{~min} /$ Woche) wurde eine professionelle Leistungsdiagnostik inkl. Spiroergometrie und Laktatstufentest nach Standards der DGP und DGSP vorgeschaltet, und somit wurden die optimalen individuellen Trainingsbereiche festgelegt.

Ergebnisse: Nach erfolgreicher Absolvierung des Trainingsprogramms konnten signifikante Verbesserungen in den Bereichen Luftnotempfinden (Borg-Summenwert: $65,7 \pm 12,2$ vs. $62,2 \pm 12,6$, Punkte, $p=0,013$ ), Körperkonstitution (BMI: 25,7 $\pm 3,3$ vs. $24,3 \pm 3,2 \mathrm{~kg} / \mathrm{m}^{2}, p=0,018$; Körperfettanteil: $24,8 \pm 5,8$ vs. $23,8 \pm 6,4 \%, p=0,043)$ sowie körperliche Leistungsfähigkeit $\left(\mathrm{VO}_{2}\right.$ bei $4 \mathrm{mmol} / \mathrm{l} \mathrm{Lak}$ tat: $24,2 \pm 6,9$ vs. $26,5 \pm 7,6 \mathrm{ml} / \mathrm{min} / \mathrm{kg}, p<0,01$; Leistung bei $4 \mathrm{mmol} / \mathrm{l}$ Laktat: Laufen/Walking $(\mathrm{n}=14)+1,1 \mathrm{~km} / \mathrm{h}, p=0,018$ und Rad/Ergometer $(\mathrm{n}=17)+8,7$ Watt, $p=0,019)$ verzeichnet werden. Diese positiven Entwicklungen zeigten sich auch in der psychischen und körperlichen Lebensqualitätssteigerung (körperliche Summenskala +9,7 Punkte, psychische Summenskala $+4,5$ Punkte), erhoben mittels SF-36-Fragebogen.

\section{Abstract \\ $\nabla$}

Background: In the process of medical rehabilitation muscular endurance training is the main focus. Unfortunately, outpatient rehabilitation opportunities are limited and specialized pulmonary exercise groups ("lung sport groups") rarely available. Therefore we developed an outpatient endurance sports program for patients with respiratory diseases and evaluated its effectiveness. Methods: In this feasibility study 31 patients ( $50 \pm 15$ years) with diverse respiratory diseases were included. By professional functional exercise testing (incl. CPET and lactate measurement according to the standards of DGP and DGSP) the patients optimal training zone was determined and an individualized 12 week lasting aerobic endurance training with $\geq 3$ sessions of $20-60 \mathrm{~min} /$ week realized.

Results: After completion of the exercise training program a significant improvement in dyspnoea (Borg-Scale: $65.7 \pm 12.2$ vs. $62.2 \pm 12.6, p=0.013$ ), body constitution (BMI: $25.7 \pm 3.3$ vs. $24.3 \pm 3.2$ $\mathrm{kg} / \mathrm{m}^{2}, p=0.018$; portion of body fat: $24.8 \pm 5.8$ vs. $23.8 \pm 6.4 \%, p=0.043)$ as well as physical capacity $\left(\mathrm{VO}_{2}\right.$ at $4 \mathrm{mmol} / \mathrm{l}$ Laktat: $24.2 \pm 6.9$ vs. $26.5 \pm 7.6$ $\mathrm{ml} / \mathrm{min} / \mathrm{kg}, p<0.01$; performance at $4 \mathrm{mmol} / 1$ Laktat: running/walking $(\mathrm{n}=14)+1.1 \mathrm{~km} / \mathrm{h}, p=0.018$ and biking/bicycle ergometer $(n=17)+8.7$ Watt, $p=0.019)$ was recorded. These positive developments were also observed in mental and physical quality of life (quality of life questionnaire SF-36: physical score +9.7 points, mental score +4.5 points).

Conclusion: The evaluated exercise program can easily be trained by the patient in a self-dependent setting and was seen to be an effective sports medical treatment in patients with diverse pulmonary diseases. 
Schlussfolgerung: Das vorgestellte ambulante Ausdauersportprogramm kann als gut durchführbar angesehen werden und erwies sich auch in eigenverantwortlicher Durchführung nach qualifizierter Anleitung als eine effektive sporttherapeutische Maßnahme bei Patienten mit unterschiedlichen pneumologischen Erkrankungen.

\section{Einleitung}

\section{$\nabla$}

Patienten mit pulmonalen Erkrankungen leiden regelhaft unter Luftnot, sind rasch müde und körperlich erschöpft. Daher meiden die meisten Patienten körperliche Anstrengungen [1,2]. Die zunehmende körperliche Inaktivität führt zu einem Verlust an Muskelmasse $[3,4]$. Außerdem kommt es durch die sinkende Ausdauerbelastung zu einer immer stärkeren Umstellung des muskulären Energiestoffwechsels vom aeroben auf den anaerben Stoffwechsel [5,6]. Die Folge ist eine erhöhte Atemarbeit bei körperlicher Belastung, da für die anearobe Energiegewinnung (Glykolyse mit erhöhter Laktatproduktion) pro Leistungseinheit wesentlich mehr ventiliertes Atemminutenvolumen benötigt wird als für den aeroben Stoffwechsel der oxidativen Phosphorylierung. Diese muskuläre Dekonditionierung mündet oft in einem erheblichen Verlust an Lebensqualität. In Anbetracht der aktuell vorliegenden Datenlage besteht längst kein Zweifel mehr daran, dass die Steigerung der körperlichen Aktivität neben der medikamentösen Standardtherapie ein wesentliches Therapieziel bei chronischen Erkrankungen der Atmungsorgane darstellen muss [7-12]. Es wird daher explizit empfohlen, dass körperliches Training integrativer Bestandteil des Langzeitmanagements sein soll und der behandelnde Arzt darauf hinwirken muss, dass der Patient regelmäßig geeignete Maßnahmen des körperlichen Trainings ergreift. Leider endet ein z. B. im Rahmen einer stationären Rehabilitationsmaßnahme begonnenes körperliches Training oft aufgrund eines fehlenden adäquaten Sportangebotes im ambulanten Bereich [13]. Des Weiteren ist aus trainingswissenschaftlicher und sportmedizinischer Sicht ein über das in der Regel nur 1 bis maximal $2 \times$ pro Woche stattfindende Lungensportangebot hinausgehendes regelmäßiges Training mit mindestens 3 Trainingseinheiten pro Woche wünschenswert [14-16]. Auch in den ATS-ERS-Empfehlungen [17] zur pneumologischen Rehabilitation wird eine Trainingsfrequenz von mindestens 3 Trainingseinheiten pro Woche empfohlen.

So wurde ein Ausdauersportprogramm mit pneumologischer und sportmedizinischer Begleitung als ambulante trainingstherapeutische Maßnahme entwickelt. Körperliche Funktionen und Belastbarkeit pneumologischer Patienten variieren intra- und interindividuell erheblich. Gleiches gilt für die Effekte von Bewegungsinterventionen. Vor diesem Hintergrund wurde der Nutzen einer sportmedizinischen Leistungsdiagnostik nach Standards der DGSP in Kombination mit den aktuellen Empfehlungen für Belastungsuntersuchungen in der Pneumologie der DGP [18] für eine optimale individuelle Trainingssteuerung und Beurteilung der Leistungsfähigkeit überprüft.

\section{Methode \\ $\nabla$}

In einem deskriptiven Ansatz wurden die Auswirkungen eines 12-wöchigen ausdauerbetonten Trainings bei pneumologischen Patienten auf Symptome, funktionelle Kapazität, Körperzusammensetzung und Lebensqualität untersucht. Die Studie fokussierte die Struktur-, Prozess- und Ergebnisqualität des Ausdauer- sportprojekts als ambulante nicht-medikamentöse Therapiemaßnahme bei Patienten mit pneumologischer Grundkrankheit. Das Ausdauersportprogramm erfolgte unter der Leitung eines Facharztes für Pneumologie mit der Zusatzbezeichnung Sportmedizin und ausreichend Erfahrung im Bereich der Leistungsdiagnostik (DGSP-qualifizierter Arzt mit entsprechendem Zertifikat).

Auf das Sportprojekt wurde mittels Aushang und Flyer in der pneumologischen Ambulanz des Helios Klinikums Emil von Behring, Trägerklinik der Lungenklinik Heckeshorn, aufmerksam gemacht und auf diese Weise potenzielle Teilnehmer rekrutiert. In das Sportprogramm eingeschlossen wurden interessierte und motivierte Patienten mit einer klinisch stabilen Lungenerkrankung. Die konsekutiv rekrutierten Patienten wurden über die diagnostischen Methoden und die sporttherapeutische Maßnahme im Rahmen des Sportprojekts aufgeklärt. Die schriftliche Zustimmung der Patienten war Voraussetzung für die Aufnahme in dieser Machbarkeitsstudie. Die Studie wurde der Ethikkommission der Charité-Universitätsmedizin Berlin vorgelegt und genehmigt (EA 2/56/11).

Zunächst wurde in einer Eingangsuntersuchung der aktuelle Gesundheitszustand überprüft und gleichzeitig eine Sporttauglichkeitsprüfung gemäß der S1-Leitlinie der Deutschen Gesellschaft für Sportmedizin und Prävention (DGSP) vorgenommen [19]. Das Untersuchungsprogramm dieser Basisuntersuchung setzte sich wie folgt zusammen:

- Anamnese (standardisierter Fragebogen für den Sportler der DGSP, standardisierter Erhebungsbogen „Sportärztlicher

Anamnese- und Untersuchungsbogen“ der DGSP)

- ausführliche körperliche Untersuchung

- apparative Untersuchungen:

- Spirometrie/Ganzkörperplethysmografie/CO-Diffusionskapazität (Master Screen ${ }^{\circledR}$ Body/Diffusion, Care Fusion, Hoechberg, Deutschland); alle gesammelten Lungenfunktionsparameter wurden als Prozent des individuellen Sollwertes (\% des Soll) angegeben, welche sich aus den Regressionsgleichungen zur Referenzwertberechnung von Quanjer u. Mitarb. [20] sowie Cotes u. Mitarb. [21] ergaben.

> Ruhe-Elektrokardiogramm (EKG)

- Ruhe-Blutgasanalyse (ABL 800 FLEX, Radiometer GmbH, Willich, Deutschland)

1. Belastungsuntersuchung: Spiroergometrie (MasterScreen ${ }^{\circledR}$ CPX, CareFusion, Hoechberg, Deutschland) mit progressivem Rampenprotokoll auf einem Fahrradergometer (Excalibur Sport, Lode B.V. Groningen, Niederlande) inkl. EKG und Blutgasanalysen unter Belastung; mit dem Ziel einer möglichst optimalen und artefaktfreien EKG-Ableitung erfolgte diese 1. Belastungsuntersuchung bei allen Patienten auf dem Fahrradergometer.

Bei erteilter Sporttauglichkeit wurden nach Ablauf von 14 Tagen in einer 2. Belastungsuntersuchung (1. Leistungstest) mittels Laktatstufentest und Spiroergometrie die individuellen ventilatorischen (VT1 + VT2) und metabolischen (Laktat-) Schwellen (LT1 + LT2) gemäß den Empfehlungen des Positionspapiers der Arbeitsgemeinschaft Spiroergometrie ermittelt [22]. Diese 2. Belastungsuntersuchung erfolgte „sportartspezifisch“: Entschied sich der Patient für ein Trainingsprogramm auf dem Fahrradergometer, absolvierte er den Laktatstufentest auf dem Fahrradergometer (Excalibur Sport, Lode B.V. Groningen, Niederlande), fiel die Wahl auf ein Lauf- bzw. Walkingtrainingsprogramm, wurde der Stufentest auf dem Laufband (pulsar ${ }^{\circledR} 3$ p, h/p/cosmos, NussdorfTraunstein, Deutschland) durchgeführt. Am Ende einer jeden Be- 
Tab. 1 Deskriptive Daten des pneumologischen Patientenkollektivs im Ausdauersportprogramm $(n=31)$.

\begin{tabular}{|c|c|c|c|}
\hline Parameter & $\begin{array}{l}\text { Weibliche } \\
\text { Patienten } \\
(n=12)\end{array}$ & $\begin{array}{l}\text { Männliche } \\
\text { Patienten } \\
\text { (n=19) }\end{array}$ & $\begin{array}{l}\text { Gesamt } \\
(n=31)\end{array}$ \\
\hline \multicolumn{4}{|l|}{ Biometrie } \\
\hline Alter (Jahre) & $50,0 \pm 3,2$ & $50,1 \pm 17,4$ & $50,4 \pm 15,1$ \\
\hline Größe (cm) & $167,3 \pm 4,0$ & $180,0 \pm 6,1$ & $175,0 \pm 8,2$ \\
\hline Gewicht $(\mathrm{kg})$ & $67,7 \pm 10,6$ & $86,1 \pm 10,8$ & $78,9 \pm 13,9$ \\
\hline $\operatorname{BMI}\left(\mathrm{kg} / \mathrm{m}^{2}\right)$ & $24,2 \pm 3,4$ & $26,6 \pm 3,0$ & $25,6 \pm 3,3$ \\
\hline FFMI & $17,8 \pm 1,5$ & $20,6 \pm 2,1$ & $19,6 \pm 2,3$ \\
\hline Körperfett (\%) & $28,9 \pm 6,9$ & $22,3 \pm 3,1$ & $24,8 \pm 5,8$ \\
\hline \multicolumn{4}{|l|}{ Lungenfunktion } \\
\hline FEV1 (\% Soll) & $80,5 \pm 15,6$ & $87,8 \pm 27,4$ & $85,0 \pm 23,5$ \\
\hline IVC (\% Soll) & $95,1 \pm 19,6$ & $98,8 \pm 16,2$ & $97,3 \pm 17,4$ \\
\hline TLC (\% Soll) & $106,7 \pm 20,4$ & $106,4 \pm 15,7$ & $106,5 \pm 17,3$ \\
\hline DLCO (\% Soll) & $61,4 \pm 23,4$ & $79,6 \pm 20,0$ & $72,5 \pm 22,8$ \\
\hline \multicolumn{4}{|l|}{ Diagnosen } \\
\hline $\begin{array}{l}\text { Chronische Sarkoidose } \\
\text { (Röntgen-Typ I/II/III/IV) }\end{array}$ & $\begin{array}{l}6(50 \%) \\
{[3 / 2 / 0 / 1]}\end{array}$ & $\begin{array}{l}9(47,4 \%) \\
{[5 / 2 / 1 / 1]}\end{array}$ & $\begin{array}{l}15(48,4 \%) \\
{[8 / 4 / 1 / 2]}\end{array}$ \\
\hline $\begin{array}{l}\text { COPD } \\
\text { (GOLD-Stadium I/II/III/IV) }\end{array}$ & $\begin{array}{l}1(8,3 \%) \\
{[1 / 0 / 0 / 0]}\end{array}$ & $\begin{array}{l}4(21,1 \%) \\
{[2 / 1 / 0 / 1]}\end{array}$ & $\begin{array}{l}5(16,1 \%) \\
{[3 / 1 / 0 / 1]}\end{array}$ \\
\hline Asthma bronchiale & $1(8,3 \%)$ & $1(5,3 \%)$ & $2(6,5 \%)$ \\
\hline Lungenkarzinom & $1(8,3 \%)$ & $2(10,5 \%)$ & $3(9,7 \%)$ \\
\hline $\begin{array}{l}\text { Lungenarterienembolie } \\
\text { (CTEPH) }\end{array}$ & $0(0 \%)$ & $1(5,3 \%)$ & $1(3,2 \%)$ \\
\hline $\begin{array}{l}\text { Interstitielle Lungen- } \\
\text { erkrankung (ILE) }\end{array}$ & $3(25 \%)$ & $2(10,5 \%)$ & $5(16,1 \%)$ \\
\hline
\end{tabular}

FFMI = Fettfreie-Masse-Index.

Tab.2 Ableitung der Trainingszonen mit relativer Orientierung an der 2. Laktatschwelle (LT2), der 1. ventilatorischen Schwelle (VT1) und dem subjektiven Luftnot-/Anstrengungsempfinden (Borg-Skala) am Beispiel eines Patienten (gewählte Ausdauersportart: Laufen).

\begin{tabular}{|lllll|}
\hline Bezeichnung & $\begin{array}{l}\text { Erholung } \\
\text { (REKOM) }\end{array}$ & $\begin{array}{l}\text { Grundlage } \\
\text { (GA1) }\end{array}$ & $\begin{array}{l}\text { Aufbau } \\
\text { (GA2) }\end{array}$ & $\begin{array}{l}\text { Entwick- } \\
\text { lung (EB) }\end{array}$ \\
\hline $\begin{array}{l}\text { Prozentbereich } \\
\text { (LT2) }\end{array}$ & $55-70 \%$ & $70-85 \%$ & $85-100 \%$ & $100-110 \%$ \\
\hline $\begin{array}{l}\text { Prozentbereich } \\
\text { (VT1) }\end{array}$ & $85-100 \%$ & $100-115 \%$ & $115-130 \%$ & $130-140 \%$ \\
\hline $\begin{array}{l}\text { Intensität } \\
\text { Borg-Skala }\end{array}$ & $9-10$ & $11-12$ & $13-14$ & $15-16$ \\
\hline $6-20]$ & $106-121$ & $121-137$ & $137-153$ & $153-163$ \\
\hline $\begin{array}{l}\text { Herzfrequenz } \\
(1 / \text { min) }\end{array}$ & mäßig & mittel & hoch \\
\hline $\begin{array}{l}\text { Geschwindigkeit } \\
\text { (km/h) }\end{array}$ & $4,4-6,1$ & $6,1-7,9$ & $7,9-9,6$ & $9,6-10,8$ \\
\hline
\end{tabular}

REKOM = Regenerations- und Kompensationstraining, GA1 = Grundlagenausdauer 1, $\mathrm{GA2}=$ Grundlagenausdauer $2, \mathrm{~EB}=$ Entwicklungsbereich.

lastungsstufe erfolgte eine Selbsteinschätzung der Belastungsluftnot mittels modifizierter Borg-Skala (RPE) [23-25]. Aus den Ergebnissen des 1. Leistungstests (Laktatschwellen LT1+2, ventilatorische Schwellen VT1+2, Einschätzung des Luftnotempfindens nach Borg) wurden die persönlichen Trainingsbereiche abgeleitet $(\bullet$ Tab.2) und ein 12-Wochen-Trainingsplan mit $\geq 3$ Trainingseinheiten à 20-60 min pro Woche erstellt (wahlweise Fahrradergometertraining oder Laufen/Walking) ( $\bullet$ Tab.3 und - Tab.4). Das Training auf dem Fahrradergometer wurde entweder daheim oder im Sportstudio durchgeführt. Das Lauf- und Walkingtraining fand in der Regel im Freien statt, wahlweise auf einem Laufband im Sportstudio. Sämtliche Trainingseinheiten erfolgten in Eigenregie und wurden vom Patienten in einem persönlichen Trainingstagebuch protokolliert. Aus den Trainingsplänen konnten die Patienten die Trainingsintensität und die Trainingsdauer entnehmen. Für sämtliche Fragen rund um das Training stand der Studienleiter telefonisch zur Verfügung.

Bei allen Patienten wurde vor Beginn und am Ende des 12-wöchigen Ausdauersportprogramms eine Bestimmung der Körperzusammensetzung (Differenzierung des Körpergewichts in Muskelund Fettmasse sowie Wasseranteil) mittels Bioimpedanzmessung (Nutribox, Data Input GmbH, Darmstadt, Deutschland) und eine Befragung mit dem SF-36-Fragebogen vorgenommen. Die abschließende 3. Belastungsuntersuchung (2. Leistungstest) zur Überprüfung der veränderten Leistungsfähigkeit erfolgte unmittelbar nach Absolvierung des 12-Wochen-Trainingsplans und wurde nach dem gleichen Untersuchungsprotokoll wie beim 2. Untersuchungstermin durchgeführt.

Die Auswertung aller Daten wurde mit dem Statistikprogramm $\mathrm{IBM}^{\circledR}{ }^{\text {SPSS }}{ }^{\circledR}$ Statistics 19 für Windows vorgenommen. Bei der deskriptiven Statistik wurden die kontinuierlichen Variabeln als Mittelwerte \pm Standardabweichung angegeben. Eventuelle Mittelwertunterschiede zweier abhängiger Gruppen (AssessmentErgebnis in den Dimensionen Luftnotempfinden, Körperzusammensetzung, Leistungsfähigkeit und Lebensqualität vor dem Ausdauersportprogramm vs. Assessment-Ergebnis nach dem Ausdauersportprogramm) wurden mittels Wilcoxon-Test für Paardifferenzen ermittelt. Grundsätzlich wurde das Signifikanzniveau eines Ergebnisses bei einem $p<0,05$ als signifikant bewertet.

\section{Ergebnisse}

$\nabla$

Von den ursprünglich 38 Patienten zu Studienbeginn erschienen 3 nicht zur Abschlussuntersuchung, 2 Patienten brachen das Training vorzeitig ab, und 2 Patienten ließen mehrere Trainingseinheiten ausfallen. Zur Auswertung gelangten somit insgesamt 31 Patienten (12 weiblich, 19 männlich), die das 12-wöchige Ausdauersportprogramm mit mindestens 3 Trainingseinheiten pro Woche und eigenständiger Protokollierung erfolgreich absolviert hatten. Das mittlere Alter betrug 50,4 $\pm 15,1$ Jahre. Die deskriptiven Daten, die Lungenfunktionsparameter und die verschiedenen Krankheitsbilder des konsekutiv eingeschlossenen Patientenkollektivs sind $\bullet$ Tab. 1 zu entnehmen. In der Eingangsuntersuchung zeigte sich lungenfunktionsanalytisch eine weite Spanne bei den dynamischen und statischen Lungenvolumina (FEV1 $27,5-134,7 \%$ des individuellen Sollwertes, IVC $62,3-135,6 \%$ des individuellen Sollwertes, TLC $71,4-147,7 \%$ des individuellen Sollwertes) sowie bei der Gasaustauschsituation (DLCO 34,0$112,9 \%$ des individuellen Sollwertes, DLCO/VA 36,4-122,3\% des individuellen Sollwertes). In der Studie waren Patienten mit obstruktiver Ventilationsstörung, restriktiver Ventilationsstörung und Perfusions-/Diffusionsstörung vertreten.

Die Effekte des ambulanten Sportprogramms wurden auf die 4 Dimensionen

1. Luftnot

2. körperliche Leistungsfähigkeit

3. Körperzusammensetzung und

4. Lebensqualität

untersucht.

Beim Luftnotempfinden konnte anhand der Borg-Skala eine signifikante Reduktion der subjektiv empfundenen Luftnot aufgezeigt werden. Dafür wurde der Borg-Summen-Score (Summe 
Tab.3 Exemplarischer 12-Wochen-Trainingsplan (gewählte Ausdauersportart: Laufen/Walken).

\begin{tabular}{|c|c|c|c|c|c|c|}
\hline $\begin{array}{l}\text { (Laufen/ Walken) } \\
\text { Start am 04.03.13 }\end{array}$ & 1./2./3. Woche & $\begin{array}{l}\text { 4. Woche } \\
\text { "Ruhe“ }\end{array}$ & 5./6./7. Woche & $\begin{array}{l}\text { 8. Woche } \\
\text { „Ruhe“ }\end{array}$ & 9./10./11. Woche & $\begin{array}{l}\text { 12. Woche } \\
\text { „Ruhe“ }\end{array}$ \\
\hline Mo. & Ruhetag & Ruhetag & Ruhetag & Ruhetag & Ruhetag & Ruhetag \\
\hline Di. & $\begin{array}{l}\text { GA1 } \\
\text { 20/25/30 min }\end{array}$ & $\begin{array}{l}\text { REKOM } \\
20 \mathrm{~min}\end{array}$ & $\begin{array}{l}\text { GA1 } \\
30 / 35 / 40 \mathrm{~min}\end{array}$ & $\begin{array}{l}\text { REKOM } \\
20-30 \text { min }\end{array}$ & $\begin{array}{l}\text { GA1 } \\
40 / 45 / 50 \mathrm{~min}\end{array}$ & $\begin{array}{l}\text { REKOM } \\
30 \mathrm{~min}\end{array}$ \\
\hline Mi. & Ruhetag & Ruhetag & Ruhetag & Ruhetag & Ruhetag & Ruhetag \\
\hline Do. & $\begin{array}{l}\text { Ein/Aus GA1 } \\
\text { je } 10 \text { min, } \\
\text { „Kurz-Intervalle“ } \\
4 \times / 5 \times / 6 \times 30 \text { sec EB, } \\
\text { dazw. } 3 \text { min REKOM }\end{array}$ & $\begin{array}{l}\text { REKOM } \\
20 \mathrm{~min}\end{array}$ & $\begin{array}{l}\text { Ein/Aus GA1 } \\
\text { je } 10 \text { min, } \\
\text { "Crecendo-Lauf“ } \\
\text { GA1 bis GA2 } \\
\text { 10/15/20 min }\end{array}$ & $\begin{array}{l}\text { REKOM } \\
20-30 \mathrm{~min}\end{array}$ & $\begin{array}{l}\text { Ein/Aus GA1 } \\
\text { je } 10 \text { min, } \\
\text { "Lang-Intervalle“ } \\
2 \times / 3 \times / 4 \text { x } 5 \text { min GA2, } \\
\text { dazw. } 3 \text { min REKOM }\end{array}$ & $\begin{array}{l}\text { REKOM } \\
30 \mathrm{~min}\end{array}$ \\
\hline Fr. & Gymnastik & Gymnastik & Gymnastik & Gymnastik & Gymnastik & Gymnastik \\
\hline Sa. & $\begin{array}{l}\text { GA1 } \\
30 / 35 / 40 \mathrm{~min}\end{array}$ & $\begin{array}{l}\text { GA1 } \\
30 \mathrm{~min}\end{array}$ & $\begin{array}{l}\text { GA1 } \\
40 / 45 / 50 \mathrm{~min}\end{array}$ & $\begin{array}{l}\mathrm{GA} 1 \\
30-40 \mathrm{~min}\end{array}$ & $\begin{array}{l}\text { GA1 } \\
50 / 55 / 60 \mathrm{~min}\end{array}$ & $\begin{array}{l}\text { GA1 } \\
40 \mathrm{~min}\end{array}$ \\
\hline So. & Ruhetag & Ruhetag & Ruhetag & Ruhetag & Ruhetag & Ruhetag \\
\hline
\end{tabular}

Ein = Einlaufen, Aus=Auslaufen. REKOM= Regenerations- und Kompensationstraining, GA1 = Grundlagenausdauer 1, GA2=Grundlagenausdauer 2, EB=Entwicklungsbereich.

Tab.4 Exemplarischer 12-Wochen-Trainingsplan (gewählte Ausdauersportart: Radfahren auf dem Fahrradergometer).

\begin{tabular}{|c|c|c|c|c|c|c|}
\hline $\begin{array}{l}\text { (Fahrrad-Ergometer) } \\
\text { Start am 11.03.13 }\end{array}$ & 1./2./3. Woche & $\begin{array}{l}\text { 4. Woche } \\
\text { "Ruhe“ }\end{array}$ & 5./6./7. Woche & $\begin{array}{l}\text { 8. Woche } \\
\text { "Ruhe“ }\end{array}$ & 9./10./11. Woche & $\begin{array}{l}\text { 12. Woche } \\
\text { "Ruhe“ }\end{array}$ \\
\hline Mo. & $\begin{array}{l}\text { GA1 } \\
20 / 25 / 30 \mathrm{~min}\end{array}$ & $\begin{array}{l}\text { REKOM } \\
20 \mathrm{~min}\end{array}$ & $\begin{array}{l}\text { GA1 } \\
30 / 35 / 40 \mathrm{~min}\end{array}$ & $\begin{array}{l}\text { REKOM } \\
20-30 \text { min }\end{array}$ & $\begin{array}{l}\text { GA1 } \\
40 / 45 / 50 \mathrm{~min}\end{array}$ & $\begin{array}{l}\text { REKOM } \\
30 \mathrm{~min}\end{array}$ \\
\hline Di. & Ruhetag & Ruhetag & Ruhetag & Ruhetag & Ruhetag & Ruhetag \\
\hline Mi. & $\begin{array}{l}\text { Ein/Aus GA } 1 \\
\text { je } 10 \text { min, } \\
\text { „Kurz-Intervalle“ } \\
4 \times / 5 \times / 6 \times 30 \text { sec EB, } \\
\text { dazw. } 3 \text { min REKOM }\end{array}$ & $\begin{array}{l}\text { REKOM } \\
20 \mathrm{~min}\end{array}$ & $\begin{array}{l}\text { Ein/Aus GA1 } \\
\text { je } 10 \text { min, } \\
\text { "Crecendo-Fahrt “ } \\
\text { GA1 bis GA2 } \\
10 / 15 / 20 \text { min }\end{array}$ & $\begin{array}{l}\text { REKOM } \\
20-30 \text { min }\end{array}$ & $\begin{array}{l}\text { Ein/Aus GA1 } \\
\text { je } 10 \text { min, } \\
\text { "Lang-Intervalle“ } \\
2 \times / 3 \times / 4 \times 5 \text { min GA2, } \\
\text { dazw. } 3 \text { min REKOM }\end{array}$ & $\begin{array}{l}\text { REKOM } \\
30 \mathrm{~min}\end{array}$ \\
\hline Do. & Ruhetag & Ruhetag & Ruhetag & Ruhetag & Ruhetag & Ruhetag \\
\hline Fr. & Gymnastik & Gymnastik & Gymnastik & Gymnastik & Gymnastik & Gymnastik \\
\hline Sa. & $\begin{array}{l}\text { GA1 } \\
30 / 35 / 40 \mathrm{~min}\end{array}$ & $\begin{array}{l}\text { REKOM } \\
30 \mathrm{~min}\end{array}$ & $\begin{array}{l}\text { GA1 } \\
40 / 45 / 50 \mathrm{~min}\end{array}$ & $\begin{array}{l}\text { REKOM } \\
30-40 \text { min }\end{array}$ & $\begin{array}{l}\text { GA1 } \\
50 / 55 / 60 \mathrm{~min}\end{array}$ & $\begin{array}{l}\text { GA1 } \\
40 \mathrm{~min}\end{array}$ \\
\hline So. & Ruhetag & Ruhetag & Ruhetag & Ruhetag & Ruhetag & Ruhetag \\
\hline
\end{tabular}

Ein $=$ Einfahren, Aus = Ausfahren. REKOM = Regenerations- und Kompensationstraining, GA1 = Grundlagenausdauer 1, GA2=Grundlagenausdauer 2, EB =Entwicklungsbereich.

der Borg-Werte, die während der stufenförmigen Belastung am Ende der 3-minütigen Belastungsstufe erfragt wurden) aus der 2. Belastungsuntersuchung (1. Leistungstest mit Laktatstufentest vor Aufnahme des Trainings) mit der 3. Belastungsuntersuchung (2. Leistungstest mit Laktatstufentest nach Absolvierung des 12Wochen-Trainingsprogramms) verglichen ( 65,7 vs. $62,2, p=0,013$ ) (৫ Abb. 1).

Das Ausmaß der körperlichen Leistungsfähigkeit wurde durch das 12-wöchige Sportprogramm deutlich verbessert. Dies zeigte sich einerseits in einer signifikanten Verbesserung der Sauerstoffaufnahme bei $4 \mathrm{mmol} / \mathrm{l}$ Laktat $(24,2$ vs. $26,5, p<0,01)$ $(\bullet$ Abb. 2), anderseits in einer signifikanten Verbesserung der erbrachten Leistung bei $4 \mathrm{mmol} / \mathrm{l}$ Laktat $(+1,1 \mathrm{~km} / \mathrm{h}, p<0,018 \mathrm{bzw}$. $+8,7$ Watt, $p=0,019)(\odot$ Abb.3).

Durch das körperliche Training erreichte das initial tendenziell übergewichtige Patientenkollektiv eine Normgewichtigkeit $(25,7$ vs. $24,3 \mathrm{~kg} / \mathrm{m}^{2}, p=0,018$ ) ( $\odot$ Abb.4) und verlor signifikant an Körperfett ( 24,8 vs. $23,8 \%, p=0,043)$ ( $\bullet$ Abb. 5 ) mit positivem Einfluss auf den Muskelaufbau („fettfreier Massen-Index“ $=$ FFMI: 19,55 vs. $19,67, p=0,42$ ).

Bei der standardisierten Erfassung der beiden Grunddimensionen körperliche und psychische Gesundheit mittels eines krankheitsübergreifenden Messinstrumentes (SF-36-Fragebogen) offenbarte sich eine signifikant positive Entwicklung auf die subjektiv empfundene Gesundheit im Sinne einer verbesserten Lebensqualität (körperliche Summenskala: +9,7 Punkte, $p<0,0001$, SES= 0,81 , SRM 1,10; psychische Summenskala: $+4,5$ Punkte, $p<0,001$, $\mathrm{SES}=0,52, \mathrm{SRM}=0,82$ ).

\section{Diskussion}

Ein regelmäßiges Bewegungstraining sollte neben einer optimalen medikamentösen Therapie integraler Bestandteil der Behandlung von pneumologischen Erkrankungen sein. Es kann dazu beitragen, Einschränkungen der körperlichen Aktivität, denen ein ungünstiger Einfluss auf die Prognose zukommt [26-30], langfristig entgegenzuwirken.

Das präsentierte Sportprogramm richtet sich an pneumologische Patienten, die zu einer Krankheitseinsicht gekommen sind und die Bereitschaft mitbringen, eine Lebensstiländerung inklusive Aufnahme regelmäßiger sportlicher Aktivitäten vorzunehmen. Da nicht alle Patienten mit einer Lungensportgruppe (räumlich und inhaltlich) erreicht werden können, wurde dieses Sportprogramm mit einem individualisierten Trainingskonzept in Eigenregie entwickelt. Die eher atypische Zusammensetzung des Patientenkollektivs in dieser Studie ergab sich aus den besonderen lokalen Gegebenheiten, da die Rekrutierung der Patienten in der 

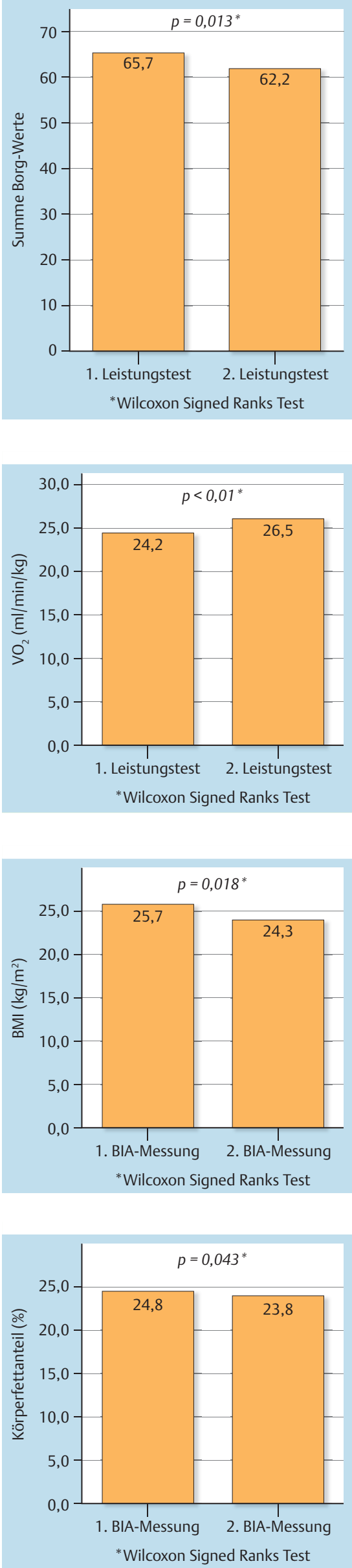

Abb. 1 Effekte des 12-wöchigen aeroben Ausdauertrainings auf das Luftnotempfinden bei körperlicher Belastung (Borg-Skala).

Abb.2 Effekte des 12-wöchigen aeroben Ausdauertrainings auf die Sauerstoffaufnahme $\left(\mathrm{VO}_{2}\right)$ bei $4 \mathrm{mmol} / \mathrm{I} \mathrm{Lak-}$ tat.

Abb. 4 Effekte des 12-wöchigen aeroben Ausdauertrainings auf den Body-Mass-Index $\left(\mathrm{kg} / \mathrm{m}^{2}\right)$.

Abb.5 Effekte des 12-wöchigen aeroben Ausdauertrainings auf den Körperfettanteil (\%). BIA= Bioelektrische Impedanz Analyse.

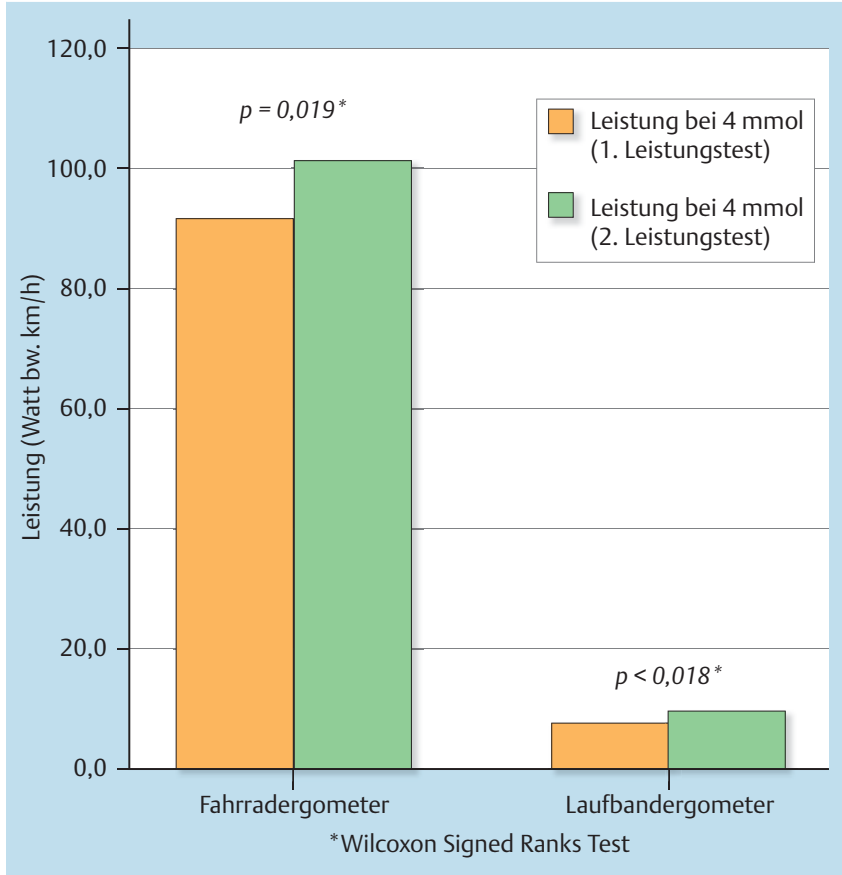

Abb.3 Effekte des 12-wöchigen aeroben Ausdauertrainings auf die Leistung bei $4 \mathrm{mmol} / \mathrm{I}$ Laktat: Laufen $(n=14):+1,1 \mathrm{~km} / \mathrm{h}$; $\operatorname{Rad}(n=17):+8,7$ Watt absolut bzw. $+0,11$ Watt $/ \mathrm{kg}$.

pneumologischen Spezialambulanz der Lungenklinik Heckeshorn stattfand. Dies stellt sicherlich eine gewisse Limitation der Studie hinsichtlich der Übertragbarkeit auf das übliche Diagnosespektrum z.B. einer Lungenfacharztpraxis dar. Es ist aber davon auszugehen, dass sich auch in anderen ambulanten pneumologischen Einrichtungen eine Vielzahl von Patienten für ein solches Sportprogramm findet und unabhängig von der Grunderkrankung und dem Schweregrad der Erkrankung von einem auf ihre individuellen körperlichen Fähigkeiten zugeschnittenen Trainingsplan profitieren wird. Weitere multizentrische Studien mit Einschluss von Patienten aus mehreren pneumologischen Praxen und Ambulanzen sollten diese Hypothese belegen können.

Für Studienzwecke erfolgte bei allen in die Studie eingeschlossenen Patienten eine sehr umfangreiche (sportmedizinische) Diagnostik. In der täglichen Praxis kann sich die Diagnostik für die Überprüfung der Sporttauglichkeit und Ermittlung der optimalen Trainingszonen an den Empfehlungen der Gesellschaft für Sportmedizin und Prävention e.V. und der fachärztlichen Einschätzung des behandelnden Pneumologen orientieren. So wird sich bei den meisten Patienten ein risikoarmes und dennoch effektives Trainingsprogramm auch mit deutlich geringerem diagnostischen Aufwand erstellen lassen. Einige Krankenkassen erstatten ihren Mitgliedern bereits zumindest einen Teil der Kosten für eine sportmedizinische Diagnostik. Basis dafür sind Verträge mit der Deutschen Gesellschaft für Sportmedizin und Prävention (DGSP).

Die vorgestellte Machbarkeitsstudie konnte zeigen, dass bewährte diagnostische Methoden aus der Sportmedizin auch gut bei pneumologischen Patienten anzuwenden sind und sich dadurch die optimalen Trainingszonen für ein gesundheitsorientiertes Sportangebot ableiten lassen. Dafür war keine körperliche Ausbelastung notwendig. Die Orientierung an den Schwellenwerten aus der Leistungsdiagnostik (LT1 + 2, VT1 + 2) und am subjektiven Luftnot- bzw. Anstrengungsgrad (Borg-Skala) ermög- 
licht eine praktikable Steuerung der gewünschten Trainingsintensitäten. Als Steuergrößen dienen Herzfrequenz (Pulsmesser), Borg-Skala und messbare Belastungsintensitäten (Wattzahl, Geschwindigkeit). Auf dieser Grundlage ist ein strukturiertes und individualisiertes Ausdauertraining mit selbständiger Kontrolle durch den Patienten möglich, das sich in dieser Form schließlich als effektive nicht-medikamentöse Therapiemaßnahme erwies. Bereits ein 12-wöchiges aerobes Ausdauertraining mit $\geq 3$ Einheiten à 20-60 min pro Woche (vornehmlich im Grundlagenausdauerbereich 1) konnte signifikante Verbesserungen in allen 4 untersuchten Bereichen (Luftnotempfinden, körperliche Leistungsfähigkeit, Körperzusammensetzung, Lebensqualität) bewirken. Wenn Lungenerkrankte ihre große, in der täglichen Alltagsbelastung am häufigsten benötigte Muskulatur (hauptsächlich große Beinmuskulatur) regelmäßig einem wohldosierten Ausdauertraining unterziehen, erfolgt durch Adaptationsprozesse auf zellulärer Ebene die gewünschte Umstellung des muskulären Energiestoffwechsels auf eine zunehmende oxidative Phosphorylierung (aerober Stoffwechsel). In der Folge kann eine körperliche Leistung mit einer wesentlich niedrigeren ventilatorischen Antwort erbracht werden. In enger Abstimmung mit dem behandelnden Pneumologen ermittelt die sportärztliche Diagnostik unter Berücksichtigung der besonderen Voraussetzungen bei pneumologischen Patienten die individuelle Leistungsfähigkeit, leitet Empfehlungen zur Trainingssteuerung ab und hilft im Bedarfsfall, Bedenken hinsichtlich einer Überforderung auszuräumen. Langfristige ambulante Sportprogramme mit individueller Eigenaktivität und fachlich qualifizierter Anleitung gemäß dem hier vorgestellten Modell können helfen, die Lücken im Angebot von sporttherapeutischen Maßnahmen zu schließen und die oftmals nur medikamentös geprägte Therapie chronisch lungenkranker Patienten um eine wichtige Therapiesäule flächendeckend zu ergänzen.

\section{Unterstützung}

Diese Studie wurde durch die Stiftung Oskar Helene-Heim, Berlin, und die Helios Kliniken GmbH unterstützt.

\section{Interessenkonflikt}

Die Autoren geben an, dass kein Interessenkonflikt besteht.

\section{Literatur}

1 Waschki B, Spruit MA, Watz $H$ et al. Physical activity monitoring in COPD: compliance and asociations with clinical characteristics in a multicenter study. Respir Med 2012; 106: 522 - 530

2 Van Remoortel H, Hornikx M, Demeyer H et al. Daily physical activity in subjects with newly diagnosed COPD. Thorax 2013; 68: 962 - 963

3 Shrikrishna D, Patel M, Tanner RJ et al. Quadriceps wasting and physical inactivity in patients with COPD. Eur Respir J 2012; 40: 1115-1122

4 Seymour JM, Spruit MA, Hopkinson NS et al. The prevalence of quadriceps weakness in COPD and the relationship with disease severitiy. Eur Respir J 2010; 36: 81 - 88

5 Lazaar AL, Greenhaff PL. Impaired muscle mitochondrial densitiy and/ or function: a COPD-specific mitochondropathy or simply deconditioning? Eur Respir J 2012; 40: 1070 -1071
6 Natanek SA, Gosker HR, Slot IG et al. Pathways associated with reduced quadriceps oxidative fibres and endurance in COPD. Eur Respir J 2013; 41: $1275-1283$

7 Gloeckl R, Marinov B, Pitta F. Practical recommendations for exercise training in patients with COPD. Eur Respir Rev 2013; 22: 178-186

8 Kenn K, Gloeckl R, Behr J. Pulmonary rehabilitation in patients with idiopathic pulmonary fibrosis - a review. Respiration 2013; 86: 89-99

9 Löllgen $H$. Bedeutung und Evidenz der körperlichen Aktivität zur Prävention und Therapie von Erkrankungen. Dtsch Med Wochenschr 2013; 138: 2253-2259

10 Halle M, Heitmann RH, Kenn $k$ et al. Bedeutung und Methodik von körperlichem Training bei COPD. Pneumologie 2008; 62: 209-221

11 Koehler U, Koehler KI, Vogelmeier C et al. Ambulanter Rehabilitationsport bei Patienten mit schwerer COPD - nur in Eigenregie möglich? Pneumologie 2010; 64: 194-195

12 Holland AE, Wadell K, Spruit MA. How to adapt the pulmonary rehabilitation programme to patients with chronic respiratory disease other than COPD. Eur Respir Rev 2013; 23: 577-586

13 Kenn K, Heinzelmann I. Pulmonary rehabilitation. Dtsch Med Wochenschr 2012; 137: 1575-1578

14 Dickhut $H H$, Löllgen $H$. Trainingsberatung für Sporttreibende. Dtsch Aerzteblatt 1996; 93: 939-943

15 American College of Sports Medicine. Guidelines for graded exercise testing and exercise prescription. 2nd ed. Philadelphia: Lea \& Febiger; 1980

16 Powell KE, Paluch AE, Blair SN. Physical activity for health: What kind? How much? How intense? On top of what? Annu Rev Public Health 2011; 32: 349-365

17 Spruit MA, Sing SJ, Carvey C et al. An Official American Thoracic Society/ European Respiratory Society Statement: key concepts and advances in pulmonary rehabilition - An executive summary. Am J Respir Crit Care Med 2013; 188: 13-64

18 Meyer FJ, Borst MM, Buschmann HC et al. Belastungsuntersuchung in der Pneumologie, Empfehlungen der Deutschen Gesellschaft für Pneumologie und Beatmungsmedizin e.V. Pneumologie 2013; 67: 16 - 34

19 DGSP. Leitlinie Vorsorgeuntersuchung im Sport. Freiburg: 2007

20 Quanjer PH, Tammeling GJ, Cotes JE et al. Lung volumes and forced ventilatory flows. Report Working Party Standardization of Lung Function Tests, European Community for Steel and Coal. Official Statement of the European Respiratory Society. Eur Respir J 1993 (Suppl. 16): 5-40

21 Cotes JE, Chinn DJ, Quanjer PH et al. Standardization of the measurement of transfer factor (diffusing capacity). Eur Respir J 1993 (Suppl. 16): $41-52$

22 Westhoff $M$, Rühle $K H$, Greiwing $A$ et al. Ventilatorische und metabolische (Laktat-)Schwellen, Positionspapier der Arbeitsgemeinschaft Spiroergometrie. Dtsch Med Wochenschr 2013; 138: 275-280

23 Borg G. Anstrengungsempfinden und körperliche Aktivität. Dtsch Aerzteblatt 2004; 101: 1016-1021

24 Löllgen H, Ulmer HV. Das „Gespräch“ während der Ergometrie: Die Borg-Skala. Dtsch Aerzteblatt 2004; 101: 1014-1015

25 Löllgen H. Standards der Sportmedizin - Das Anstrengungsempfinden (RPE, Borg-Skala). Deutsche Zeitschrift für Sportmedizin 2004; 11: $299-300$

26 Erikssen $G$, Liestoel $K$, Bjoernholt $J$ et al. Changes in physical fitness and changes in mortality. Lancet 1998; 352: 759-762

27 Garcia-Aymerich J, Farrero E, Felez MA et al. Risk factors of readmission to hospital for a COPD exazerbation: a prospective study. Thorax 2003; 58: $100-105$

28 Revitt $O$, Sewell L, Morgan MD et al. A short out-patient pulmonary rehabilitation programme reduces readmission following a hospitalisation for an exacerbation of COPD. Respirology 2013; 18: 1063-1068

29 Waschki B, Kirsten A, Holz O et al. Physical activity is the strongest predictor of all-cause mortality in patients with COPD: a prospective cohort study. Chest 2011; 140: 331 - 342

30 Wen CP, Wai JP, Tsai MK et al. Minimum amount of physical activity for reduced mortality and extended life expetancy: a prospective cohort study. Lancet 2011; 378: $1244-1253$ 